\title{
A novel iteration class for solution of nonlinear equation
}

\author{
Hamideh Eskandari \\ Department of Mathematics, Payame Noor University, Isfahan, I. R. Iran
}

\section{Email address:}

h_eskandari@pnu.ac.ir

\section{To cite this article:}

Hamideh Eskandari. A Novel Iteration Class for Solution of Nonlinear Equation. American Journal of Applied Mathematics. Vol. 2, No. 5, 2014, pp. 186-190. doi: 10.11648/j.ajam.20140205.16

\begin{abstract}
In this paper, one of the most fundament problems in numerical analysis has designed that it is found roots of equation $\mathrm{f}(\mathrm{x})=0$ with variable $\mathrm{x}$. In different articles and books, many several of methods exist for solving non algebra equations. Here, we present a class of Halley method and Chebyshev method from second derivatives for solving non algebra equations. In fact, it can be said that the best and the most convenient solution is the Newton method. We have this new method called class of Halley-Chebyshev method that this method also has second derivatives.
\end{abstract}

Keywords: Non Algebra Equation, Newton Method, Hybrid Method, Halley Method, Chebyshev Method

\section{Introduction}

We consider iterative methods to find a single root of a non algebra equation

$$
f(x)=0
$$

The roots of the equation (1) or solution of equation (1) is a usual problem in applied mathematics and we are looking for a simple numerical method by which these non algebra equation to solve.

To determine the root of an equation with good accuracy it is often necessary that we know approximate of roots in little interval that root belong to interval.

Function $f(x)$ usually has a continuous order first derivative is at least and an initial estimate of the root often have. Numerical methods for solution of equation (1) of the initial estimate uses and it generates sequence with better estimates give of the root. These kind methods are iterative methods. One of the iterative methods is Newton-Raphson method (or simply Newton's method) and it is one of the most useful and most well known algorithms that it depends on continuous $f^{\prime}(x)$ and $f^{\prime \prime}(x)$.

To use this method, it should be to estimate the near of the root target on available. Let $x_{0}$ is approximate $\alpha$ and $h$ is the difference between them so that we have $\alpha=x_{0}+h$.

If we obtain $h$ and then we add to $x_{0}$, we attain to $\alpha$. By using of Taylor expansion, we have

$$
0=f(\alpha)=f\left(x_{0}+h\right)=f\left(x_{0}\right)+h f^{\prime}\left(x_{0}\right)+\frac{h^{2}}{2} f^{\prime \prime}(\eta)
$$

Where $\eta$ is between $\alpha$ and $x_{0}$. And where $h$ is very tiny. Hence, we can ignore from last sentence of above expansion, i.e. $0 \cong f\left(x_{0}\right)+h f^{\prime}\left(x_{0}\right)$. So

$$
h \cong-\frac{f\left(x_{0}\right)}{f^{\prime}\left(x_{0}\right)}
$$

So if we add $-\frac{f\left(x_{0}\right)}{f^{\prime}\left(x_{0}\right)}$ to $x_{0}$ then we obtain approximate of $\alpha$ and we call it $x_{1}$, i.e.

$$
x_{1}=x_{0}-\frac{f\left(x_{0}\right)}{f^{\prime}\left(x_{0}\right)}
$$

And thus we achieved the following formula

$$
x_{n+1}=x_{n}-\frac{f\left(x_{n}\right)}{f^{\prime}\left(x_{n}\right)}
$$

This formula is a convergence of order two. This formula can be found in many books such as [5, 6, and 8].

Another method in [3] which is called "hybrid method" is offered by the following iteration formula

$$
x_{n+1}=\frac{-B \pm \sqrt{B^{2}-4 A C}}{2 A}
$$

Where

$$
A=f^{\prime \prime}\left(x_{n}\right) \quad \text { and } \quad B=6 f^{\prime}\left(x_{n}\right)-2 f^{\prime \prime}\left(x_{n}\right) x_{n}
$$


$C=6 f\left(x_{n}\right)-6 f^{\prime}\left(x_{n}\right) x_{n}+f^{\prime \prime}\left(x_{n}\right) x_{n}^{2}$.

Which this formula has a better convergence than Newton's method.

In [5] and also in [6], a solution for one variable non algebra equation is presented, which through using Taylor extension this iteration formula is obtained. This iteration method is:

$$
x_{n+1}=x_{n}-\frac{f^{\prime}\left(x_{n}\right) \pm \sqrt{\left(f^{\prime}\left(x_{n}\right)\right)^{2}-2 f\left(x_{n}\right) f^{\prime \prime}\left(x_{n}\right)}}{f^{\prime \prime}\left(x_{n}\right)}
$$

This iteration formula is the third order and it is proved in [7] with this assumption $\Delta=\left(f^{\prime}\left(x_{n}\right)\right)^{2}-2 f\left(x_{n}\right) f^{\prime \prime}\left(x_{n}\right)$ for $\Delta>0$, $\Delta=0$ and $\Delta<0$, the intended answer will be reached.

Other method that we will review here is new method in [4]. If it is assumed that $x_{n+1}-x_{n}=\Delta$, then the following equation is obtained

$$
A \Delta^{2}+B \Delta+C=0
$$

In which

$$
A=(1-2 \omega) f^{\prime \prime}\left(x_{n}\right), B=2 f^{\prime}\left(x_{n}\right)
$$

$C=2 f\left(x_{n}\right)$

By solving this equation for $\Delta$ yields the following sentence is gotten

$$
\Delta=\frac{-B \pm \sqrt{B^{2}-4 A C}}{2 A}
$$

This method has iteration formula

$$
\begin{aligned}
& x_{n+1}=x_{n} \\
& -\frac{f^{\prime}\left(x_{n}\right) \pm \sqrt{\left(f^{\prime}\left(x_{n}\right)\right)^{2}-2(1-2 \omega) f\left(x_{n}\right) f^{\prime \prime}\left(x_{n}\right)}}{(1-2 \omega) f^{\prime \prime}\left(x_{n}\right)}
\end{aligned}
$$

Which (5) equation is the new iteration formula and $\omega$ is considered as an arbitrary parameter, and $0 \leq \omega \leq 1$.

Other method such as Halley and Chebyshev methods, for solving non algebra equations (1) exists, which we explain in the next section.

\section{Halley and Chebyshev Methods}

In this paper, we consider several iterative methods for finding roots of non algebra equations (1). In [1], Halley method is completely explained and by using modification Newton method, this method have obtained. In the method that called Halley method, we have iteration formula

$$
x_{n+1}=x_{n}-\frac{f\left(x_{n}\right)}{\frac{1}{2} f^{\prime \prime}\left(x_{n}\right)\left(y_{n}-x_{n}\right)+f^{\prime}\left(x_{n}\right)}
$$

Where

$$
y_{n}=x_{n}-\frac{f\left(x_{n}\right)}{f^{\prime}\left(x_{n}\right)}
$$

And this sentence is Newton iteration. So we have

$$
x_{n+1}=x_{n}-\frac{f\left(x_{n}\right) f^{\prime}\left(x_{n}\right)}{\left(f^{\prime}\left(x_{n}\right)\right)^{2}-\frac{1}{2} f\left(x_{n}\right) f^{\prime \prime}\left(x_{n}\right)}
$$

This iterative method is contains second order derivative.

Also, if an iteration method, named Chebyshev method, in [2] is explained, and we have:

$$
x_{n+1}=x_{n}-\left(1+\frac{1}{2} \frac{f^{\prime \prime}\left(x_{n}\right) f\left(x_{n}\right)}{\left(f^{\prime}\left(x_{n}\right)\right)^{2}}\right) \frac{f\left(x_{n}\right)}{f^{\prime}\left(x_{n}\right)}
$$

This sentence is also the second order derivative.

Because both iteration of method second order derivative is obtained the new method presented as follows.

\section{Halley-Chebyshev Method}

If we combine the iteration formulas (1) and (2) together, then we get a new iteration formulas and this iteration formula is also the second order derivative.

If we multiply Halley method iteration (7) in $\omega$ and multiply Chebyshev method iteration $(8)$ in $(1-\omega)$ where $\omega$ is considered as an arbitrary parameter and $0 \leq \omega \leq 1$, then we have a sentence iteration that is convex compound of Halley-Chebyshev method. So

$$
\begin{gathered}
\omega x_{n+1}-(1-\omega) x_{n+1}=\omega\left(x_{n}-\frac{f\left(x_{n}\right) f^{\prime}\left(x_{n}\right)}{\left(f^{\prime}\left(x_{n}\right)\right)^{2}-\frac{1}{2} f\left(x_{n}\right) f^{\prime \prime}\left(x_{n}\right)}\right) \\
-(1-\omega)\left(x_{n}-\left(1+\frac{1}{2} \frac{f^{\prime \prime}\left(x_{n}\right) f\left(x_{n}\right)}{\left(f^{\prime}\left(x_{n}\right)\right)^{2}}\right) \frac{f\left(x_{n}\right)}{f^{\prime}\left(x_{n}\right)}\right)
\end{gathered}
$$

After simplifying, we obtain this sentence

$$
\begin{aligned}
x_{n+1} & =x_{n} \\
- & -\left(\begin{array}{l}
\omega \frac{f\left(x_{n}\right) f^{\prime}\left(x_{n}\right)}{\left(f^{\prime}\left(x_{n}\right)\right)^{2}-\frac{1}{2} f\left(x_{n}\right) f^{\prime \prime}\left(x_{n}\right)} \\
+(1-\omega)\left(1+\frac{1}{2} \frac{f^{\prime \prime}\left(x_{n}\right) f\left(x_{n}\right)}{\left(f^{\prime}\left(x_{n}\right)\right)^{2}}\right) \frac{f\left(x_{n}\right)}{f^{\prime}\left(x_{n}\right)}
\end{array}\right)
\end{aligned}
$$

Because derivative is linear, so above iteration also is the second order derivative. Thus we see that we can find different formulas with different $\omega$ s and $0 \leq \omega \leq 1$.

Now, for comparison this iteration formula (9) with the other methods, we bring several examples in the next section.

\section{Numerical Examples}

In here, we have real several methods of non algebra equation that we compare them with together in tables. 


\subsection{Example 1}

Consider the equation $f(x)=\sin x-\cos x+x-2$. This function has a single root with accurate amount 1.301766754799 . In order to find a root close to $x=1.301766754799$, we let $x_{0}=0$.

The obtained results by Newton iteration, new hybrid iteration [3], and Halley method [1] are presented in the under tables. (Table 1 with $\varepsilon=10^{-1000}$ )

Table 1. Newton, Hybrid and Halley methods

\begin{tabular}{|c|c|c|c|c|c|c|}
\hline \multirow{2}{*}{$\mathbf{n}$} & \multicolumn{2}{|c|}{ Newton's method } & \multicolumn{2}{|c|}{ Hybrid's method [3] } & \multicolumn{2}{|c|}{ Halley's method [1] } \\
\hline & $x_{n}$ & $f\left(x_{n}\right)$ & $x_{n}$ & $f\left(x_{n}\right)$ & $x_{n}$ & $f\left(x_{n}\right)$ \\
\hline 0 & 0. & -3 . & 0 . & -3 & 0 . & -3. \\
\hline 1 & 1.500000000000 & 0.426757784936 & 1.348469228350 & 0.103356154323 & 1.090909090909 & -0.483723071241 \\
\hline 2 & 1.293660601863 & -0.018098136173 & 1.301527415041 & -0.000533705850 & 1.300774210270 & -0.002213544659 \\
\hline 3 & 1.301756590548 & -0.000022664540 & 1.301766748823 & $-1.3325 * 10^{-8}$ & 1.301766754685 & $-2.54 * 10^{-10}$ \\
\hline 4 & 1.301766754783 & $-3.6 * 10^{-11}$ & 1.301766754798 & $-2 * 10^{-12}$ & 1.301766754799 & 0. \\
\hline 5 & 1.301766754799 & 0. & 1.301766754797 & $-4 * 10^{-12}$ & 1.301766754799 & 0 . \\
\hline 7 & & & 1.301766754802 & 0 . & & \\
\hline 8 & & & 1.301766754802 & 0. & & \\
\hline
\end{tabular}

We see that number of $\mathrm{n}$ in Halley method in Table 1 is 5, this namely that Halley method is better than Newton and Hybrid methods in Table 1.

The obtained results by the new iteration method with different $\omega$ [4] and Chebyshev method is the under tables. (Table 2 with $\varepsilon=10^{-1000}$ )

Table 2. Chebyshev and New iteration methods

\begin{tabular}{|c|c|c|c|c|c|c|}
\hline \multirow{2}{*}{$\mathbf{n}$} & \multicolumn{2}{|c|}{ New iteration method [4] with $\omega=0$} & \multicolumn{2}{|c|}{ New iteration method [4] with $\omega=0.35$} & \multicolumn{2}{|c|}{ Chebyshev's method } \\
\hline & $x_{n}$ & $f\left(x_{n}\right)$ & $x_{n}$ & $f\left(x_{n}\right)$ & $x_{n}$ & $f\left(x_{n}\right)$ \\
\hline 0 & 0. & -3 & 0. & -3 & 0. & -3 \\
\hline 1 & 1.162277660168 & -0.317262379628 & 1.361063052528 & 0.130950507660 & 1.190004278328 & -0.253281064739 \\
\hline 2 & 1.301505416663 & -0.000582762235 & 1.301355020706 & -0.000918154278 & 1.301686404186 & -0.000179170086 \\
\hline 3 & 1.301766754798 & $-2 * 10^{-12}$ & 1.301766736229 & $-4.1408 * 10^{-8}$ & 1.301766754799 & 0 \\
\hline 4 & 1.301766754798 & $-2 * 10^{-12}$ & 1.301766754797 & $-4 *^{*} 10^{-12}$ & 1.301766754799 & 0. \\
\hline 5 & & & 1.301766754799 & 0. & & \\
\hline 6 & & & 1.301766754799 & 0. & & \\
\hline
\end{tabular}

We see that number of $\mathrm{n}$ in Chebyshev method in Table 2 is 4; this namely that Chebyshev method is better than New iteration method in Table 2.

The obtained results by the Halley-Chebyshev method with different $\omega$ is the under tables. (Table 3 with $\varepsilon=10^{-1000}$ )

Table 3. Halley-Chebyshev method

\begin{tabular}{ccccccc}
\hline & \multicolumn{2}{c}{ Halley-Chebyshev method with $\omega=0.3$} & \multicolumn{2}{c}{ Halley-Chebyshev method with $\omega=0.5$} & \multicolumn{2}{c}{ Halley-Chebyshev method with $\omega=0.9$} \\
\cline { 2 - 7 } & $x_{n}$ & $f\left(x_{n}\right)$ & $x_{n}$ & $f\left(x_{n}\right)$ & $x_{n}$ & $f\left(x_{n}\right)$ \\
\hline 0 & 0. & -3. & 0. & -3. & 0. & -3. \\
1 & 1.377272727273 & 0.166287499442 & 1.295454545455 & -0.014088982866 & 1.131818181818 & -0.388010096836 \\
2 & 1.301085536615 & -0.001519159506 & 1.301763650303 & -0.000006922487 & 1.300947700674 & -0.001826581724 \\
3 & 1.301766703980 & $-1.13317 * 10^{-7}$ & 1.301766754798 & $-2 . * 10^{-12}$ & 1.301766744251 & $-2.3520^{*} 10^{-8}$ \\
4 & 1.301766754799 & 0. & 1.301766754798 & $-2 . * 10^{-12}$ & 1.301766754799 & 0. \\
5 & 1.301766754799 & 0. & & & 1.301766754799 & 0. \\
\hline
\end{tabular}

We see that number of $\mathrm{n}$ in Halley-Chebyshev method with $\omega=0.5$ in Table 3 is 4 ; this namely that Halley-Chebyshev method is better than Newton and Hybrid methods with $\omega=0.5$ in Table 3 .

So we can change $\omega$ and thus we could have better methods than the other methods.

\subsection{Example 2}

Consider the equation $f(x)=\ln \left(x^{2}+3 x+3\right)-e^{-x}+4$. This function has a single root with accurate amount -1.322660733 .
In order to find a root close to $x=-1.322660733$, we let $x_{0}=0$.

The obtained results by Newton iteration, new hybrid iteration [3], and Halley method [1] are presented in the under tables. (Table 4 with $\varepsilon=10^{-1000}$ )

We see that number of $\mathrm{n}$ in Halley method in Table 4 is 4; this namely that Halley method is better than Newton and Hybrid methods in Table 4.

The obtained results by the new iteration method with different $\omega$ [4] and Chebyshev method is the under tables. (Table 5 with $\varepsilon=10^{-1000}$ ) 
Table 4. Newton, Hybrid and Halley methods

\begin{tabular}{|c|c|c|c|c|c|c|}
\hline \multirow[b]{2}{*}{$\mathbf{n}$} & \multicolumn{2}{|c|}{ Newton's method } & \multicolumn{2}{|c|}{ Hybrid's method [3] } & \multicolumn{2}{|c|}{ Halley's method [1] } \\
\hline & $x_{n}$ & $f\left(x_{n}\right)$ & $x_{n}$ & $f\left(x_{n}\right)$ & $x_{n}$ & $f\left(x_{n}\right)$ \\
\hline 0 & 0. & 4.098612289 & 0. & -1.720430475 & 0. & 4.098612289 \\
\hline 1 & -2.049306144 & -3.712069860 & -1.720430475 & -1.811841097 & -1.217576882 & 0.434394506 \\
\hline 2 & -1.496745739 & -0.754796143 & -1.344747543 & -0.093267606 & -1.322595980 & 0.000272431 \\
\hline 3 & -1.328106587 & -0.022932939 & -1.322714814 & -0.000227534 & -1.322660733 & 1. $* 10^{-9}$ \\
\hline 4 & -1.322665665 & -0.000020749 & -1.322660735 & $-7 . * 10^{-9}$ & -1.322660733 & 1. * $10^{-9}$ \\
\hline 5 & -1.322660733 & $1 *^{*} 10^{-9}$ & -1.322660732 & $5 * 10^{-9}$ & & \\
\hline 6 & -1.322660733 & $1 *^{*} 10^{-9}$ & -1.322660734 & $-3 * * 10^{-9}$ & & \\
\hline 7 & & & -1.322660733 & $1 *^{*} 10^{-9}$ & & \\
\hline 8 & & & -1.322660733 & 1.*10 $0^{-9}$ & & \\
\hline
\end{tabular}

Table 5. Chebyshev and New iteration methods

\begin{tabular}{|c|c|c|c|c|c|c|}
\hline \multirow{2}{*}{$\mathbf{n}$} & \multicolumn{2}{|c|}{ New iteration method $[4]$ with $\omega=0$} & \multicolumn{2}{|c|}{ New iteration method [4] with $\omega=0.35$} & \multicolumn{2}{|c|}{ Chebyshev's method } \\
\hline & $x_{n}$ & $f\left(x_{n}\right)$ & $x_{n}$ & $f\left(x_{n}\right)$ & $x_{n}$ & $f\left(x_{n}\right)$ \\
\hline 0 & 0. & 4.098612289 & 0. & 4.098612289 & 0. & 4.098612289 \\
\hline 1 & -1.397916223 & -0.320641555 & -1.744854441 & -1.935846277 & -0.2456651932 & 3.564541148 \\
\hline 2 & -1.322634368 & 0.000110924 & -1.350276514 & -0.116722871 & -0.8838853780 & 1.701575949 \\
\hline 3 & -1.322660734 & $-3 * * 10^{-9}$ & -1.322749524 & -0.000373572 & -1.311499720 & 0.046870264 \\
\hline 4 & -1.322660733 & $1 .^{*} 10^{-9}$ & -1.322660734 & $-3 . * 10^{-9}$ & -1.322660661 & $3.04 * 10^{-7}$ \\
\hline 5 & -1.322660733 & $1 *^{*} 10^{-9}$ & -1.322660734 & $-3 . * 10^{-9}$ & -1.322660733 & $1 .^{*} 10^{-9}$ \\
\hline 6 & & & & & -1.322660733 & $1 .^{*} 10^{-9}$ \\
\hline
\end{tabular}

Table 6. Halley-Chebyshev method

\begin{tabular}{|c|c|c|c|c|c|c|}
\hline \multirow{2}{*}{ n } & \multicolumn{2}{|c|}{ Halley-Chebyshev method with $\omega=0.3$} & \multicolumn{2}{|c|}{ Halley-Chebyshev method with $\omega=0.5$} & \multicolumn{2}{|c|}{ Halley-Chebyshev method with $\omega=0.9$} \\
\hline & $x_{n}$ & $f\left(x_{n}\right)$ & $x_{n}$ & $f\left(x_{n}\right)$ & $x_{n}$ & $f\left(x_{n}\right)$ \\
\hline 0 & 0. & 4.098612289 & 0. & 4.098612289 & 0. & 4.098612289 \\
\hline 1 & - 1.799787366 & -2.222866466 & -1.633441513 & -1.385687382 & -1.300749809 & 0.091849686 \\
\hline 2 & -1.358721170 & -0.152631010 & -1.330525538 & -0.033132679 & -1.322668559 & -0.000032925 \\
\hline 3 & -1.322812038 & -0.000636596 & -1.322665877 & -0.000021641 & -1.322660733 & $1 *^{*} 10^{-9}$ \\
\hline 4 & -1.322660736 & $-1.1 * 10^{-8}$ & -1.322660733 & $1 *^{*} 10^{-9}$ & -1.322660733 & 1. $* 10^{-9}$ \\
\hline 5 & -1.322660733 & $1 . * 10^{-9}$ & -1.322660733 & $1 . * 10^{-9}$ & & \\
\hline 6 & -1.322660733 & $1 .^{*} 10^{-9}$ & & & & \\
\hline
\end{tabular}

We see that number of $\mathrm{n}$ in new iteration method with different $\omega$ in Table 5 is 5 , this namely that new iteration method with different $\omega$ is better than Chebyshev methods in Table 5.

The obtained results by the Halley-Chebyshev method with different $\omega$ is the under tables. (Table 6 with $\varepsilon=10^{-1000}$ )

We see that number of $\mathrm{n}$ in Halley-Chebyshev method with $\omega=0.9$ in Table 6 is 4 , this namely that Halley-Chebyshev method with $\omega=0.9$ is better than Newton and Hybrid methods and other methods in Table 6.

So we can change $\omega$ and thus we could have better methods than the other methods.

\section{Conclusion}

In this paper, recent iteration formula is offered to solve the roots of the nonlinear equations. Tables and results show that this method has better performance than other methods. It can be seen from the examples that the current method is enough a faster method that takes lesser number of iterations, needs lesser number of functional evaluations in final as well as in individual step as compared to the other methods.

\section{References}

[1] Jisheng Kou, Yitian Li, Xiuhua Wang, On modified Newton methods with cubic convergence, Applied Mathematics and Computation, 176 (2006) 123-127.

[2] Jisheng Kou, Yitian Li, Xiuhua Wang, A uniparametric Chebyshev-type method free from second derivatives, Applied Mathematics and Computation, 179 (2006) 296-300.

[3] Nasr-Al-Din Ide, A new hybrid iteration method for algebraic equations, Applied Mathematics and Computation, 195 (2008) $772-774$.

[4] Hamideh Eskandari, A new numerical solving method for equations of one variable, International Journal of Applied Mathematics and Computer Sciences, 5:3 (2009) 183-186.

[5] Stoer .J, Bulirsch .R, Introduction to numerical analysis, Springer-Verlag, $3^{\text {rd }}$ ed., 2002. 
[6] Hildebrand .F.B, Introduction to numerical analysis, Tata McGraw-Hill, Second edition, 1987.

[7] Fang .T, Fang .G, Lee .C.F, A new iteration method with cubic convergence to solve nonlinear algebraic equations, Applied Mathematics and Computation, 175 (2006) 1147-1155.
[8] Atkinson, Kendall E. An introduction to numerical analysis, John Wiley \& Sons, 1988. 